\title{
Green Synthesis of Gold Nanoparticles Using Origanum vulgare
}

\author{
MS Aguilar ${ }^{1 *}$, F Mares-Briones $^{1}$, SE Borjas-García ${ }^{2}$ and G Rosas ${ }^{1}$ \\ 1. Instituto de Investigación en Metalurgia y Materiales, UMSNH, edificio U, ciudad universitaria, \\ Morelia, Michoacán, MEXICO. \\ 2. Instituto de Física y Matemáticas, UMSNH, ciudad universitaria, Morelia, Michoacán, MEXICO. \\ * Corresponding author: maaguilar@umich.mx
}

In recent years, the interest of the synthesis of gold nanoparticles has increased due to its electronic, optical, chemical, physical, and catalysis properties [1,2]. Various methods have been developed for the synthesis of gold nanoparticles (AuNPs). Among them, the green process synthesis based on plant extracts is a method of great interest for being an easy, fast and friendly process with the environment. In this investigation, the green synthesis of AuNPs is presented: using gold salt $\left(\mathrm{HAuCl}_{4}\right)$ as precursor agent and extract of Origanum vulgare as reducing and stabilizing agent, the reaction was carried out at room temperature. The effect of the concentration of Origanum vulgare extract $(0.01 \mathrm{~g} / \mathrm{ml}, 0.02 \mathrm{~g} / \mathrm{ml}$ and $0.03 \mathrm{~g} / \mathrm{ml}$ ) on the morphology of AuNPs was evaluated. For this, the gold salt concentration remained constant $(3 \mathrm{mM})$. The extract of the Origanum vulgare plant contains flavonoid biomolecules, monoterpenes and polyphenols [3] acting as a reducing and stabilizing agent to reduce metal ions to nanoparticles. For the preparation of the extract, $10 \mathrm{~g}$ of the dried plant of Origanum vulgare were milled into a fine powder. $2 \mathrm{~g}$ of powder was suspended in $100 \mathrm{ml}$ of distilled water and heated at $60{ }^{\circ} \mathrm{C}$ for $30 \mathrm{~min}$, then filtered and the supernatant was used in the synthesis. For biosynthesis of gold nanoparticles, typically, $5 \mathrm{ml}$ aqueous solution of gold salt $(3 \mathrm{mM})$ was mixed with different concentrations of Origanum vulgare extract at room temperature. During the reaction, a continuously color change was observed from yellow to dark purple, indicates the formation of gold nanoparticles. After seeing the color change, the solids were collected by centrifugation at 12,000 rpm, followed by repeated washing with distilled water to remove remaining material. Different characterization techniques were used to evaluate the biosynthesis of AuNPs. UV-vis spectroscopy was used for the evaluation of particle formation. The scanning electron microscopy, as well as the transmission electron microscopy, was used to determine the size, morphology and chemical composition of the synthesized solids. X-ray diffraction analysis was carried out to determine the crystal structure. The UV-vis spectra show a peak of surface plasmon (SPR) in the range of $500 \mathrm{~nm}$ to $600 \mathrm{~nm}$. The position of SPR peak coincides with the previously reported for AuNPs. As can be seen in Fig. 1 as the concentration of Origanum vulgare extract increase, the SPR was narrower, suggesting a more significant number of nanoparticles with monodisperse sizes. The electron microscopy studies illustrate AuNPs with different morphology as shown Fig. 2a to 2c. In the Fig. 2d displays the energy dispersive spectrum analysis (EDS) corroborating the presence of $\mathrm{Au}$ in the sample. XRD patterns of the nanoparticles synthesized are shown in Fig. 3, which exhibit peaks at $38.21^{\circ}, 44.41^{\circ}, 64.62^{\circ}$ and $77.62^{\circ}$ corresponding to the (111), (200), (220) and (311) planes of the Au-FCC which matches with Joint Committee on Powder Diffraction Standards (JCPDS) data file (01-089-3697). The peaks at $28.22^{\circ}$ and $40.46^{\circ}$ are attributed to the extract-derived components. In summary, gold nanoparticles were synthesized by a biological method using the aqueous extract of Origanum vulgare as reducing and stabilizing agent. UV-vis spectroscopy showed the SPR absorption band at $570 \mathrm{~nm}$, characteristic of AuNPs. The studies by electron microscopy show Au nanoparticles with shapes of spherical, triangular and icosahedral of average size of $20 \mathrm{~nm}$ [4]. 
References:

[1] CD Pina et al., Chem. Soc. Rev. 37 (2008), p. 2077.

[2] S Alex et al., J. Nanosci. Nanotechnol 15 (2015), p. 1869.

[3] S Koldas et al., J. Sci. Food Agric. 95 (2015), p. 786.

[4] The authors acknowledge funding from Consejo Nacional de Ciencia y Tecnología (CONACyT), México.

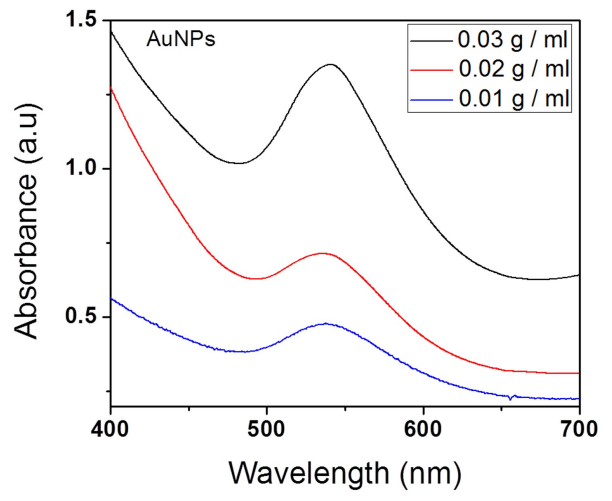

Figure 1. UV-vis spectra of AuNPs synthesized at $3 \mathrm{mM}$ of $\mathrm{HAuCl}_{4}$ and different plant extract concentration.

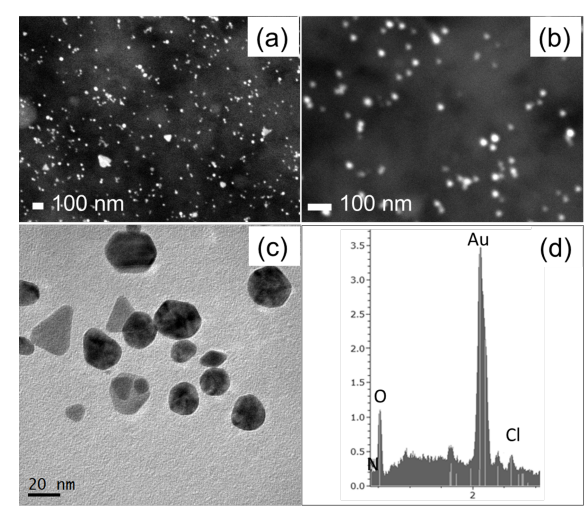

Figure 2. a and b) SEM of gold nanoparticles obtained using Origanum vulgare extract, c) bright-field TEM images of the AuNPs synthesized, and d) EDS analysis of solids obtained.

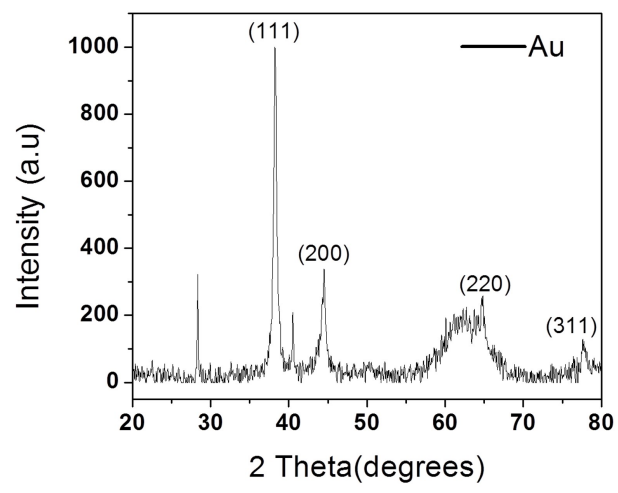

Figure 3. XRD pattern of gold nanoparticles synthesized. 